\title{
Implementation of Mobile Health Technologies in Clinical Trials of Movement Disorders: Underutilized Potential
}

\author{
Carlo Alberto Artusi ${ }^{1}$. Gabriele Imbalzano ${ }^{1}$ - Andrea Sturchio ${ }^{2}$. Andrea Pilotto ${ }^{3,4}$. Elisa Montanaro ${ }^{1}$. \\ Alessandro Padovani ${ }^{3} \cdot$ Leonardo Lopiano $^{1} \cdot$ Walter Maetzler ${ }^{5}$ • Alberto J. Espay ${ }^{2}$ (I)
}

Published online: 30 July 2020

(C) The American Society for Experimental NeuroTherapeutics, Inc. 2020

\begin{abstract}
Mobile health technologies (mHealth) are patient-worn or portable devices aimed at increasing the granularity and relevance of clinical measurements. The implementation of mHealth has the potential to decrease sample size, duration, and cost of clinical trials. We performed a review of the ClinicalTrials.gov database using a standardized approach to identify adoption in and usefulness of mHealth in movement disorders interventional clinical trials. Trial phase, geographical area, availability of data captured, constructs of interest, and outcome priority were collected. Eligible trials underwent quality appraisal using an ad hoc 5point checklist to assess mHealth feasibility, acceptability, correlation with patient-centered outcome measures, and clinical meaningfulness. A total of $29 \%(n=54 / 184)$ registered trials were using mHealth, mainly in Parkinson's disease and essential tremor (59.3\% and 27.8\%). In most cases, mHealth were used in phase 2 trials $(83.3 \%)$ as secondary outcome measures $(59.3 \%)$. Only five phase 3 trials, representing $9.3 \%$ of the total, used mHealth (1 as primary outcome measure, 3 as secondary, and 1 as tertiary). Only $3.7 \%(n=2 / 54)$ of all trials used mHealth for measuring both motor and non-motor symptoms, and $23.1 \%(n=12 /$ 52) used mHealth for unsupervised, ecologic outcomes. Our findings suggest that mHealth remain underutilized and largely relegated to phase 2 trials for secondary or tertiary outcome measures. Efforts toward greater alignment of mHealth with patientcentered outcomes and development of a universal, common-language platform to synchronize data from one or more devices will assist future efforts toward the integration of mHealth into clinical trials.
\end{abstract}

Key Words Technology $\cdot$ mobile $\cdot$ outcome measure $\cdot$ movement disorders $\cdot$ Parkinson's disease $\cdot$ tremor.

Electronic supplementary material The online version of this article (https://doi.org/10.1007/s13311-020-00901-x) contains supplementary material, which is available to authorized users.

Alberto J. Espay

alberto.espay@uc.edu

1 Department of Neuroscience "Rita Levi Montalcini", University of Torino, Torino, Italy

2 Gardner Family Center for Parkinson's disease and Movement Disorders, Department of Neurology, University of Cincinnati Academic Health Center, 260 Stetson St., Suite 2300, Cincinnati, OH 45267-0525, USA

3 Department of Clinical and Experimental Sciences, University of Brescia, Brescia, Italy

4 FERB Onlus, Ospedale S. Isidoro, Trescore Balneario, Bergamo, Italy

5 Department of Neurology, Christian-Albrechts-University of Kiel, Kiel, Germany

\section{Introduction}

The advent of a new generation of technology-based objective measures for application in health science seems particularly suitable for the field of movement disorders. These tools encompass wearable or portable devices, referred to as mobile health technologies (mHealth) (https://www.who.int/ehealth/ en/), rendering possible the quantification of many behavioral constructs of interest in a continuous, minimally invasive manner. mHealth have the potential to improve accuracy in diagnosis, monitoring, therapeutic choices, and prognosis for patients with movement disorders. Moreover, they can capture information in an ecologically valid ("natural") setting in everyday life [1]. Several portable devices have been applied so far. Inertial measurement units (IMUs), measuring linear and angular motion (e.g., accelerometers, gyroscope), are the most widespread mHealth in movement disorders. They are relatively easy to use, low-cost, and able to measure the most common constructs of interest (e.g., tremor, bradykinesia, 
dyskinesia, gait) [2]. However, other wearable technologies employed are as follows: biopotential electrode sensors, which converts ion current on the skin to electrical energy (e.g., EEG); optical sensors, which converts light energy to electrical energy (e.g., glasses); and chemical sensors, which convert chemical energy to electrical energy (electrochemical skin sensing) (Fig. 1) [3].

Despite technological advances, such as miniaturization, portability, and increased connectivity, the implementation of mHealth in clinical trials has lagged due to concerns regarding validation and acceptability, difficulties in data interpretation, unclear correlation with patient-centered outcomes (such as independence and quality of life), lack of an endorsed regulatory pathway, and application to endpoints with variable relationships with severity and disability (e.g., tremor) $[1,4,5]$.

An improvement in the accuracy, reproducibility, feasibility, and clinical meaningfulness of mHealth measures would theoretically decrease the sample size of clinical trials, shortening their duration and lowering their cost, while obtaining a comprehensive, longitudinal tracking of meaningful motor and nonmotor data at home and in community settings $[1,5$, 6]. To this end, the International Parkinson and Movement Disorders Society (MDS) Task Force on Technology has recently recommended a roadmap to overcome obstacles and improve the adoption of mHealth in the field of movement disorders [5].

Fig. 1 Examples of mHealth
To realize the clinically meaningful and cost-saving use of mHealth, it is of importance to understand the extent to which ongoing clinical trials in movement disorders are adapting the MDS Task Force objectives on the use of mHealth, with particular attention to which gaps may remain between the trials and the MDS roadmap. We, therefore, sought to analyze the current use of mHealth in ongoing clinical trials in movement disorders. Given the high number of published studies reporting on the use of mHealth in Parkinson's disease (PD), we also performed an exploratory analysis of all the constructs of interest which can be captured in a comfortable, minimally invasive manner through a smartphone or tablet and a motion sensor worn at the belt.

\section{Methods}

\section{Search of ClinicalTrials.gov}

We searched using a standardized approach the ClinicalTrials.gov database for interventional clinical trials of movement disorders using mHealth, defined as wearable or portable devices capturing data in a frequent or continuous, and minimally invasive way.

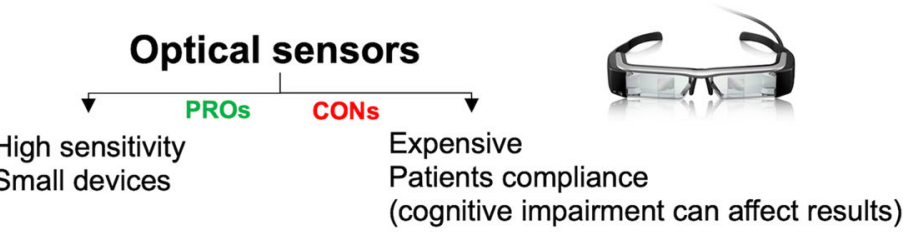

Biopotential electrode sensors

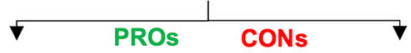

Uncomfortable, bulky

$\begin{array}{ll}\text { Pathophysiology-symptoms } & \text { Uncomfortable, bulky } \\ \text { correlation } & \text { Patients compliance }\end{array}$

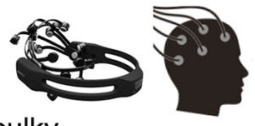

(cognitive impairment can affect results)

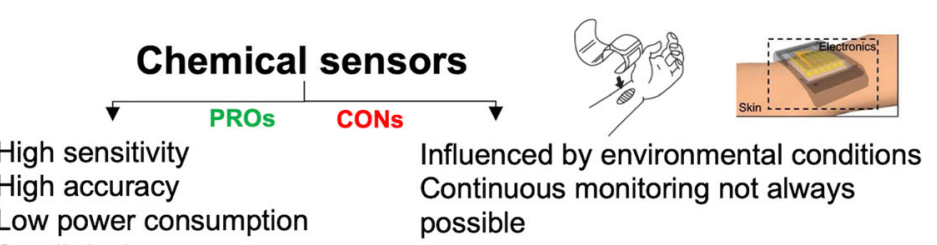

Small devices

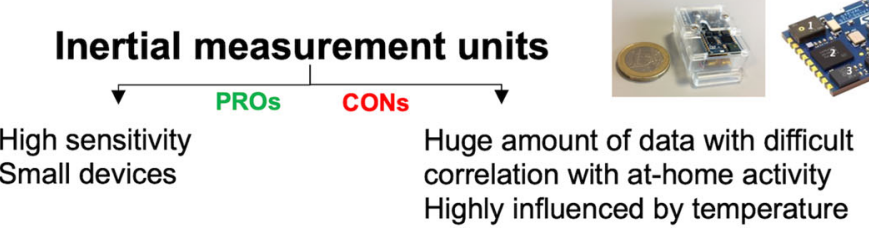




\section{Participants and Interventions}

We selected movement disorders clinical trials in phases 2, 3, or 4 , in which mHealth were employed. There were no restrictions applied to gender, age, disease duration, type of movement disorder, type of intervention, or study design. Suspended or withdrawn trials were excluded.

\section{Data Collection and Analysis}

Search Methods Relevant articles were identified through an electronic search of Clinicaltrials.gov conducted in January 2020 using the following keywords: Parkinson's disease, dystonia, tremor, chorea, and movement disorders. Duplicated studies were identified by the "ClinicalTrials.gov Identifier" code and were removed from the database.

Selection of Studies Four authors (G.I., A.P., E.M., A.S.) screened all search results to identify studies meeting the inclusion criteria, in which mHealth were employed. The outcome measures of each trial were evaluated for the presence of devices. All trials for which we ascertained the use of a technology corresponding to the definition of mHealth (as reported above) were included. When information on the technology used in the study was lacking, details were sought using email correspondence with the contact person for the corresponding trial. No limitations in dates were applied except for trials concluded before or without update after 2018.

Data Extraction and Analysis Data extracted included the following: type of movement disorder, phase of trial, status of trial, geographical area of trial, single- or multicenter, presence of a sponsor, type of mHealth, justification for using mHealth (classified as stated, derived, or unknown), and availability of data captured by mHealth. Constructs of interest captured by mHealth (e.g., tremor, bradykinesia, gait, sleep) were also collected along with information on the priority of the outcome assessed (i.e., primary, secondary or tertiary outcome measure).

In addition, each eligible trial underwent a "quality appraisal" to evaluate the way mHealth are used, and which were the pitfalls in mHealth implementation. To this end, an independent quality evaluation by two movement disorder experts was performed using an ad hoc 5-point checklist to assess the strengths and pitfalls in the application of mHealth concerning the following aspects:

1. Validation (i.e., verification of psychometric properties of the outcome measure or its correlation with other outcome measures)

2. Number of construct(s) of interest measured

3. Clinical meaningfulness of captured data (i.e., verification that significant changes of mHealth mesures were identified as important by patients, and indicate a potential change in the patient's management)

4. Formal assessment of mHealth feasibility and acceptability

5. Correlation between data captured by mHealth and patient-centered outcome measures

The quality appraisal tool was developed as a checklist of the presence of the abovementioned aspects suggested by the MDS technology task force for optimal integration of technology in clinical trials. It was not intended as a quality appraisal of the trial itself. Data are reported as the total of "yes" counts (i.e., adherence to the suggested claim) each trial reported for each of the five questions (Fig. 2).

Finally, for each trial, we evaluated whether the mHealth was used in a supervised setting (i.e., during in-clinic specific tasks), home-like environment (i.e., in-clinic, supervised multiple tasks simulating a domestic environment), or unsupervised setting (i.e., during daily life).

Descriptive statistics were used for summary measures, as appropriate.

\section{Exploratory Analysis}

As an exploratory analysis, we aimed to identify all the validated and published algorithms demonstrating to reliably assess PD motor and non-motor constructs of interest by the use of a single inertial sensor worn at the trunk or by a smartphone/tablet. To this end, we reviewed PubMed to January 2020 and also relied on a systematic review of technologies employed for the evaluation of motor and non-motor phenomena in PD [4]. Reviews on the same topic and reference lists of screened articles were checked for further relevant articles. We reported all constructs of interest for which studies validated a monitoring algorithm using an inertial sensor positioned at the trunk or a smartphone/tablet. We also verified if the device and algorithm were validated for a supervised setting, home-like environment, or unsupervised setting. Devices and algorithms validated for differential diagnoses were not considered.

\section{Results}

\section{Systematic Review of ClinicalTrials.gov}

After the exclusion of duplicates, the search of the clinicaltrials.gov database yielded 184 trials. Out of the 184 identified trials, 29\% $(n=54)$ used mHealth and underwent data extraction (Table 1 and Fig. 3). 83.3\% of included trials were phase $2(n=45), 9.3 \%$ phase $3(n=5)$, and $7.4 \%$ phase 4 $(n=4)$ (Fig. 4); 66.7\% ( $n=36)$ were single-center studies, and $40 \%(n=22)$ were sponsored by a pharma company. The 
Fig. 2 Quality assessment tool for assessing the use of mHealth in clinical trials
Quality Assessment Tool for Interventional Trials using Mobile Health Technologies

Article:

\begin{tabular}{|c|c|c|c|c|}
\hline No. & Criteria & Yes & No & $\begin{array}{l}\text { Other } \\
\text { (CD/NR/ } \\
\text { NA)* }\end{array}$ \\
\hline 1. & $\begin{array}{l}\text { Are mobile health technologies already validated? } \\
\text { (i.e., accuracy, reliability, and sensitivity data already tested and published) }\end{array}$ & $x$ & & \\
\hline 2. & $\begin{array}{l}\text { Do mobile health technologies capture } \geq 1 \text { constructs of interest, or they only } \\
\text { assess a single domain? } \\
\text { (Please, record the number of constructs of interest assessed) }\end{array}$ & $x$ & & \\
\hline 3. & $\begin{array}{l}\text { Did the measures captured by mobile health technologies prove to be clinically } \\
\text { meaningful in previous peer reviewed publications? If not, does the trial enable } \\
\text { testing whether mobile health technologies could be clinically meaningful? } \\
\text { (i.e., tested correlation between mobile health technologies and clinical endpoints) }\end{array}$ & $x$ & & \\
\hline 4. & $\begin{array}{l}\text { Were feasability and acceptability issues related to mobile health technologies } \\
\text { investigated in previous peer reviewed publications? If not, does the trial enable } \\
\text { testing feasability and acceptability issues? } \\
\text { (i.e., SUMI questionnaire or similar analyzed) }\end{array}$ & $x$ & & \\
\hline 5. & $\begin{array}{l}\text { Were associations between the employed mobile health technologies and patients } \\
\text { related outcome measures evaluated in previous peer reviewed publications? If not, } \\
\text { is it planned to evaluate these associations in the trial? } \\
\text { (i.e., association between endpoints captured by mobile health technologies and } \\
\text { impairment in activities of daily living or quality of life?) }\end{array}$ & $x$ & & \\
\hline \multicolumn{5}{|c|}{ Total number of 'Yes': } \\
\hline \multicolumn{5}{|c|}{ Additional Comments: } \\
\hline
\end{tabular}

${ }^{*} \mathrm{CD}$, cannot determine; NA, not applicable; NR, not reported

mHealth employed was specified for 27 trials; for other 6 trials, we communicated with the assigned contact to obtain the missing information; thus, $61.1 \%$ of trials $(n=33)$ underwent quality appraisal for the use of mHealth.

\section{Target Population and Outcomes}

The target population was PD in $59.3 \%$ of studies $(n=32)$, tremor in $27.8 \%$ ( $n=15$, of which 13 were essential tremor [ET], 1 tremor in fragile X-associated tremor/ataxia syndrome [FXTAS], and 1 antipsychotic-induced tremor), dystonia in $1.8 \%(n=1)$, chorea in $1.8 \%(n=1$ on Huntington disease $[\mathrm{HD}])$, and other movement disorders in $9.3 \%(n=5)$. The latter included 3 on progressive supranuclear palsy (PSP), 1 on schizophrenia (tardive dyskinesia), and 1 on FXTAS hypertension.

Concerning PD, only 2 trials included patients at any disease stage (Hoehn and Yahr stage 1 to 5); most trials included patients within specific but large stages, from newly diagnosed to advanced PD.

mHealth was used for quantifying the primary outcome in $44.4 \%$ of studies $(n=24)$, secondary outcome in $59.3 \%(n=$ $32)$, and tertiary or exploratory outcome in $11.1 \%(n=6)$. $18.5 \%$ of trials $(n=10)$ used mHealth for more than one outcome: primary + secondary in 9; secondary + tertiary in 1 (Table 1 and Fig. 4). Phase 2 trials used mHealth as primary outcome measures in $24 \%$ of cases $(n=11)$, as primary + secondary in $20 \%(n=9)$, as secondary in $40 \%(n=18)$, as secondary + tertiary in 3\% $(n=1)$, and as tertiary in $13 \%(n=$ 6); phase 3 trials used mHealth in only 1 trial as primary outcome measure, 3 as secondary, and 1 as tertiary. Of the four phase 4 trials, 3 used mHealth as primary outcome measures and 1 as secondary. From the trials disclosing this information, mHealth data was captured during specific tasks in $40.7 \%$ of studies $(n=22)$, passively in the background in $33.3 \%(n=18)$, both actively and passively in $7.4 \%(n=4)$.

The geographical areas of included studies are reported in Fig. 5.

\section{mHealth Types and Applications}

All mHealth employed are listed in Table 1 and Fig. 4. The most common were wearable motion sensors, used in $75.9 \%$ of studies $(n=41)$, and smartphone applications, used in 5.6\% $(n=3)$. mHealth were used to assess motor constructs of interest in $87 \%$ of studies $(n=47)$, non-motor constructs in 9.3\% $(n=5)$, and both motor and non-motor in $3.7 \%(n=2)$ (Table 1 and Fig. 4). The reason for using mHealth was stated or derived in $92.6 \%$ of studies $(n=50)$. Among all studies, $29.6 \%(n=16)$ allow mHealth data capture be available for the scientific community, freely or upon request. mHealth were used in a supervised setting in $71.1 \%$ of trials $(n=37)$, 


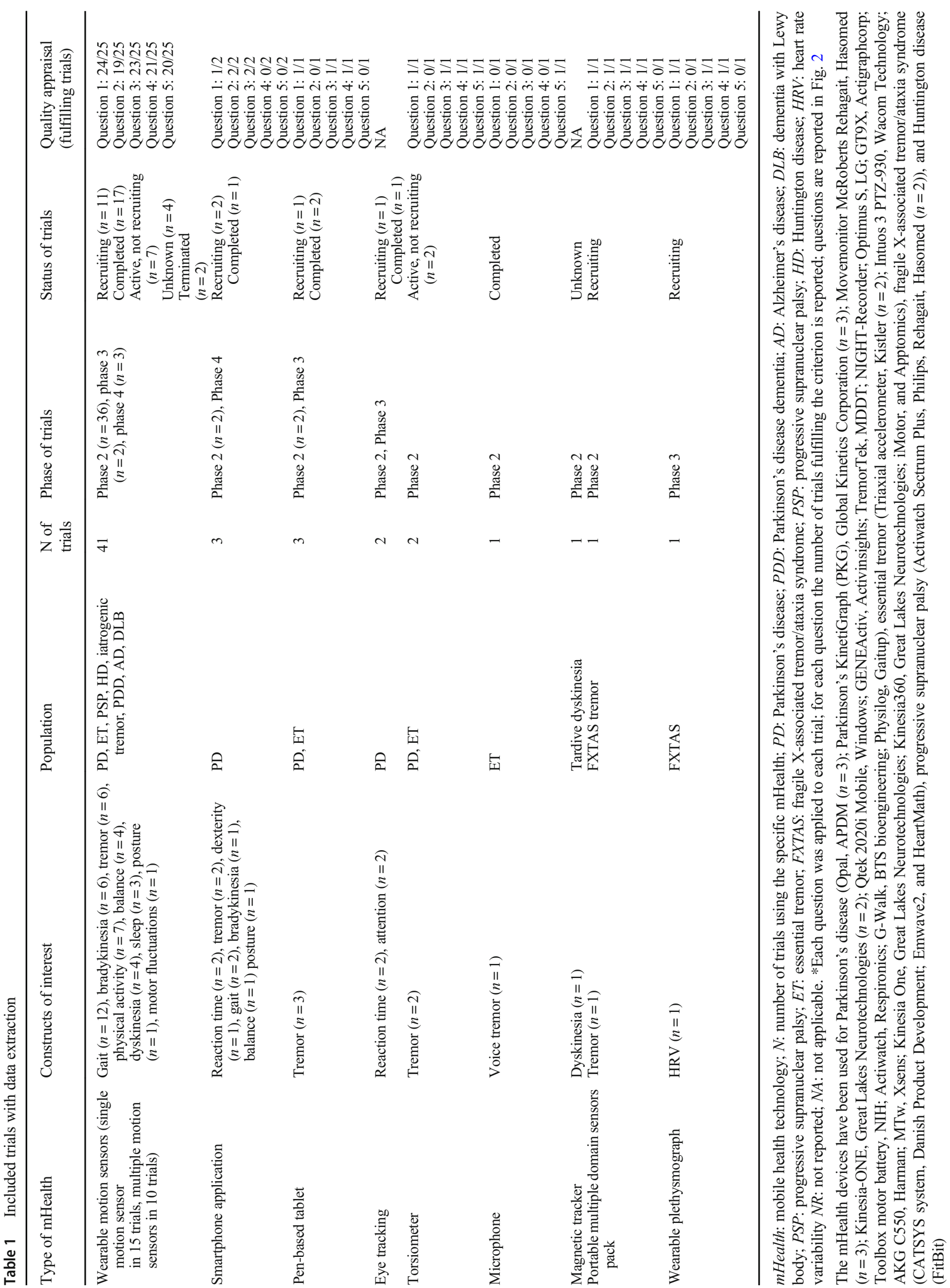


Fig. 3 Flowchart of the systematic review

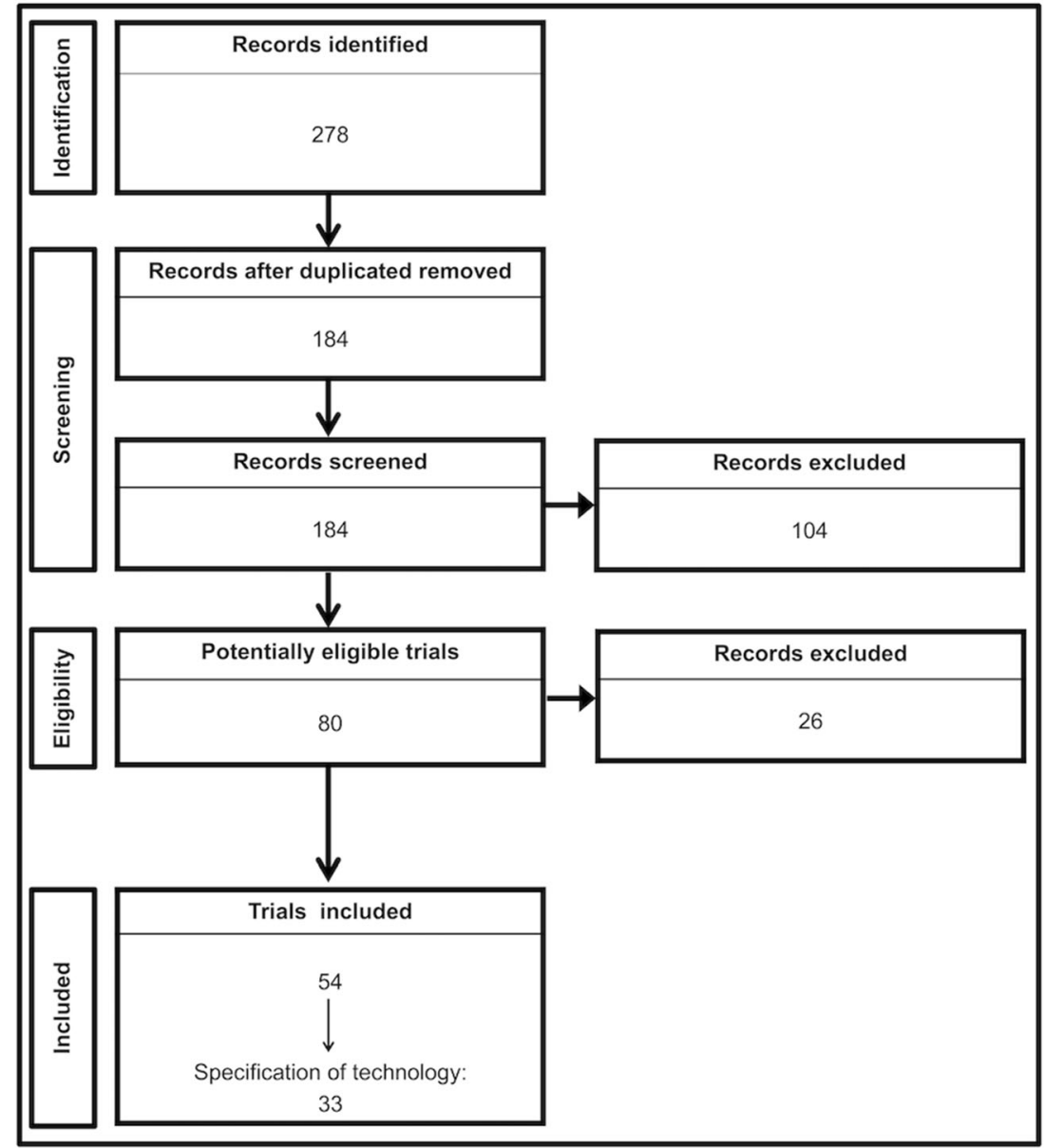

in an unsupervised setting in $23.1 \%(n=12)$, and in both supervised and unsupervised settings in $5.8 \%(n=3)$. This information was not retrievable in 2 trials.

\section{Quality Appraisal of mHealth Use}

The use of 35 devices employed in 33 trials was assessed by the quality appraisal tool. mHealth already validated in previous studies were used in $91.4 \%$ of trials $(n=32) .62 .8 \%(n=$ 22) captured multiple constructs of interest, and $37.2 \%(n=$ 13) captured a single construct of interest. Clinical meaningfulness was previously examined for $91.4 \%$ of mHealth employed $(n=32)$, and feasibility and acceptability for $80 \%$ $(n=28) .71 .4 \%(n=25)$ of mHealth employed had an established correlation with patient-related outcome measures, such as activities of daily living or quality of life (Table 1 and Fig. 6).

\section{Exploratory Analysis}

We identified 15 different constructs of interest provided by 14 studies [7-20]. Results showed that PD patients wearing an inertial sensor at the trunk while using a smartphone or tablet can be monitored with validated algorithms for a high number of constructs of interest, especially in supervised settings. In particular, tremor, bradykinesia, reaction time, dyskinesia, postural transitions, balance, gait, falls risk, freezing of gait (FoG), upper limb freezing, writing quality, turning, physical activity (number of steps), and sleep quality can be monitored in a supervised setting; dyskinesia, FoG, postural transition, turning, gait, physical activity, and falls risk in a home-like environment; and sleep quality in an unsupervised setting (Fig. 7). The timed up and go test (TUG), an assessment encompassing the gait, transfers, and turning constructs of interest, also showed to be reliable analyzed both in supervised and in home-like environment settings.

\section{Discussion}

This systematic analysis of mobile technologies in clinical trials of movement disorders showed that the use of mHealth remains limited, mostly confined to trials in $\mathrm{PD}$ and ET, and overwhelmingly used in phase 2 trials as secondary outcome measures. Most trials captured motor symptoms (mainly gait and tremor); only two used mHealth for measuring both motor 

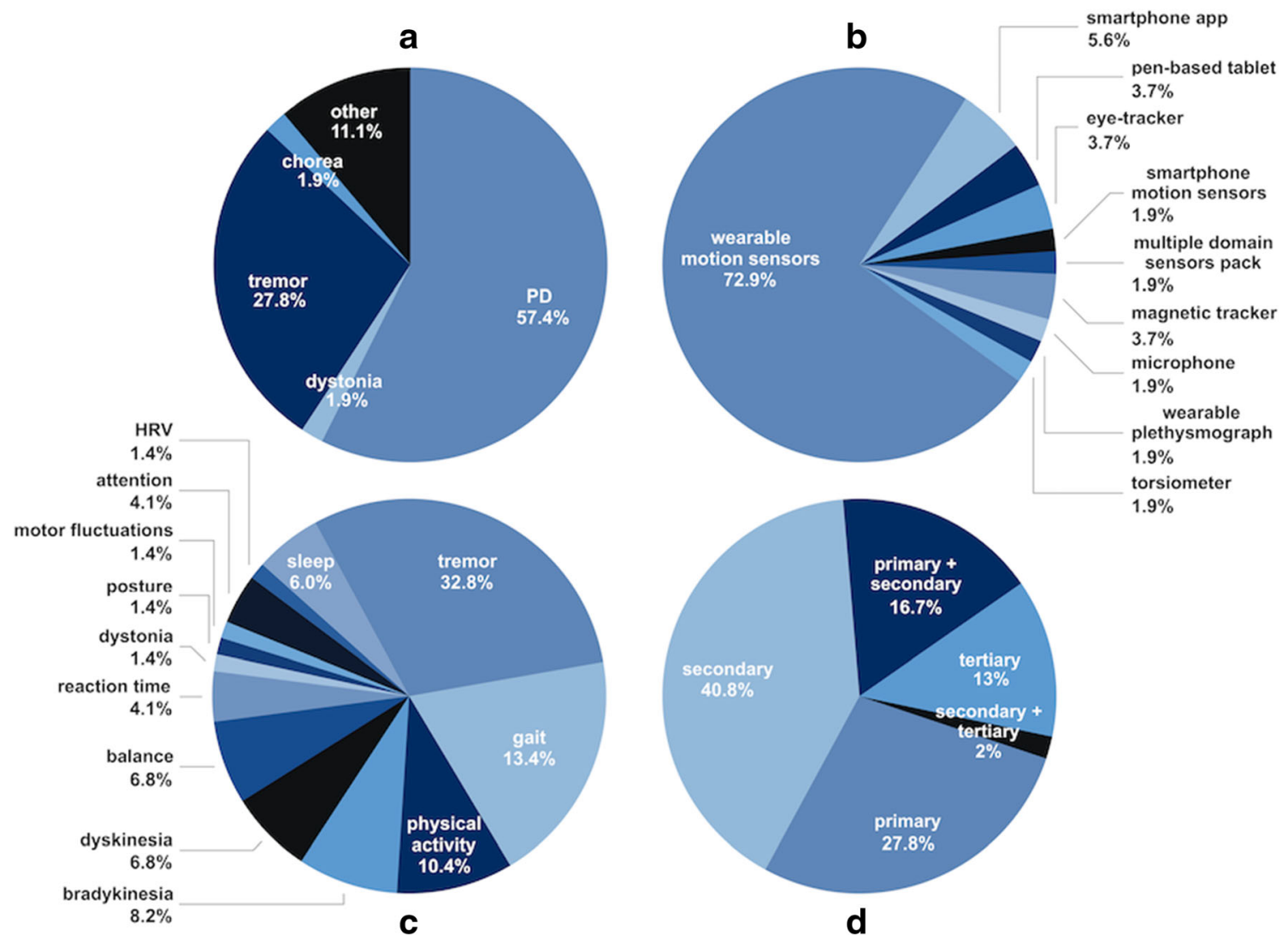

Fig. 4 Information extracted from included trials. (a) Target population; (b) category of mobile health technology (mHealth) used; (c) constructs of interest assessed by mHealth; (d) type of outcome measure for which mHealth is used; HRV: heart rate variability

and non-motor symptoms. Also, most trials used mHealth for outcomes obtained during the execution of specific tasks (i.e., supervised setting), and less than $25 \%$ of all trials used mHealth for unsupervised, ecologically relevant outcome measures.
The finding that in most trials mHealth are used for secondary or tertiary outcomes adds to the evidence that further efforts are needed to integrate mHealth as valid and reliable outcome measures into research. Another relevant aspect emerging from our review is the low number of trials using $\mathrm{mHealth}$ in unsupervised

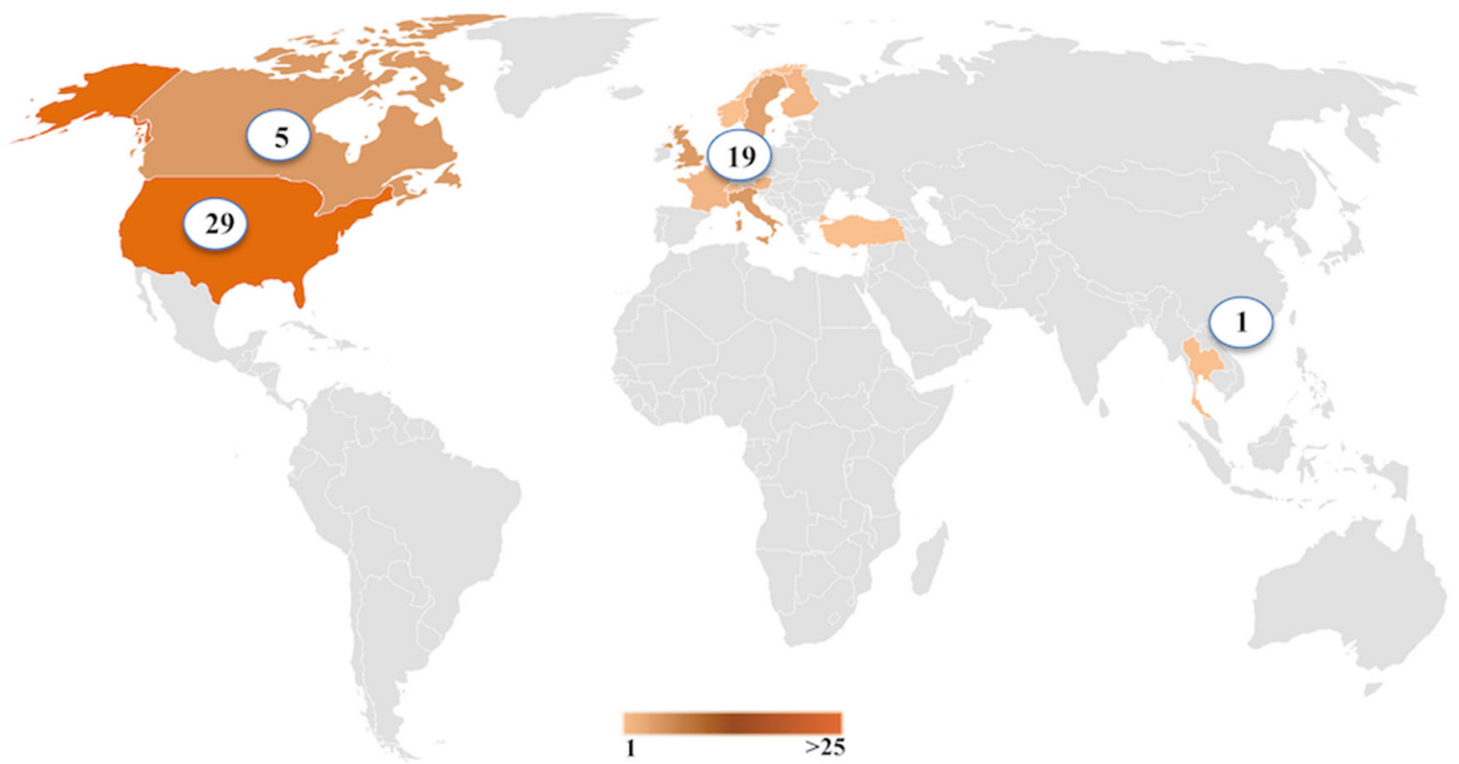

Fig. 5 Geographical areas of included trials. Numbers represent the number of trials for each specific geographic area included in the review 
Fig. 6 Quality appraisal of mobile health technologies
40

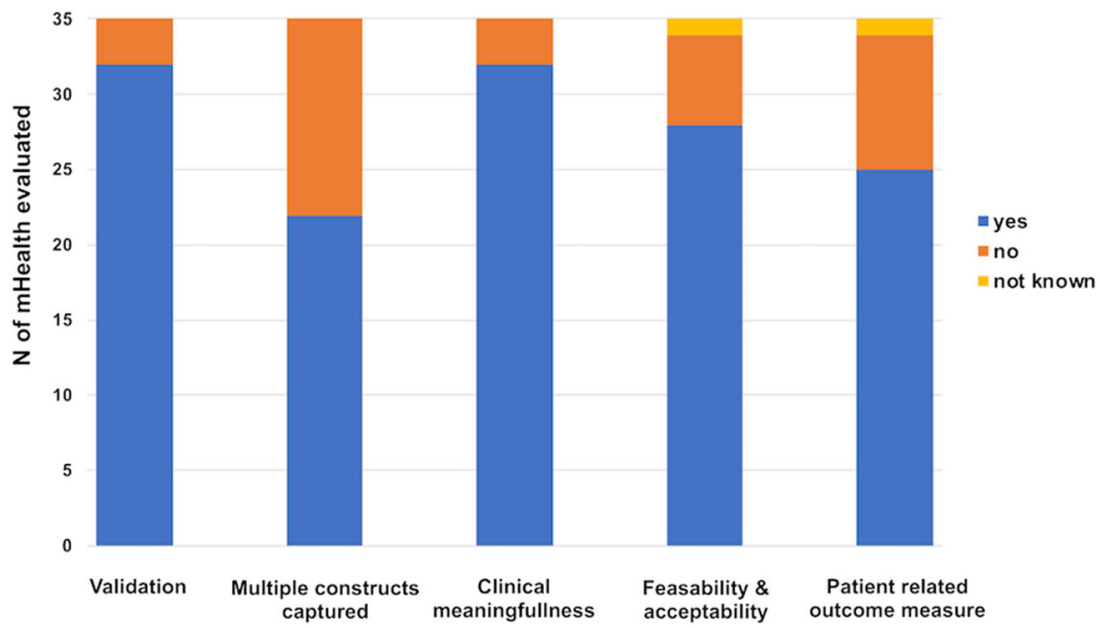

settings. In fact, the added value of unsupervised assessments has been well documented [21, 22], and an accurate ecologic analysis of outcomes should be the ultimate goal of the integration of mHealth in clinical trials, as recently highlighted by the European Medicines Agency (EMA) and US Food and Drug Administration (FDA) [23] (https://www.fda.gov/medicaldevices/digital-health/wireless-medical-devices).

Mobile technologies are accessible and affordable tools with obvious, promising usefulness in improving the monitoring of movement disorders [5, 24]. The potential of increasing the accuracy of outcome measures should reduce sample size, duration, and costs of trials [1]. Moreover, mHealth could facilitate enrolment and adherence to trials, overcoming difficulties in transportation and geographical distance as barriers for trial participation. In the last few years, there is an increased interest in the use of wearable sensors and other mHealth to replace clinical scales for monitoring the disease course and assessing the therapeutic effect of patients
Fig. 7 Constructs of interest potentially to be captured by a single inertial sensor worn at the trunk and a smartphone. TUG: timed up and go test. Physical activity is intended as the total number of steps during a task (supervised), a time frame (homelike), or daily (unsupervised)

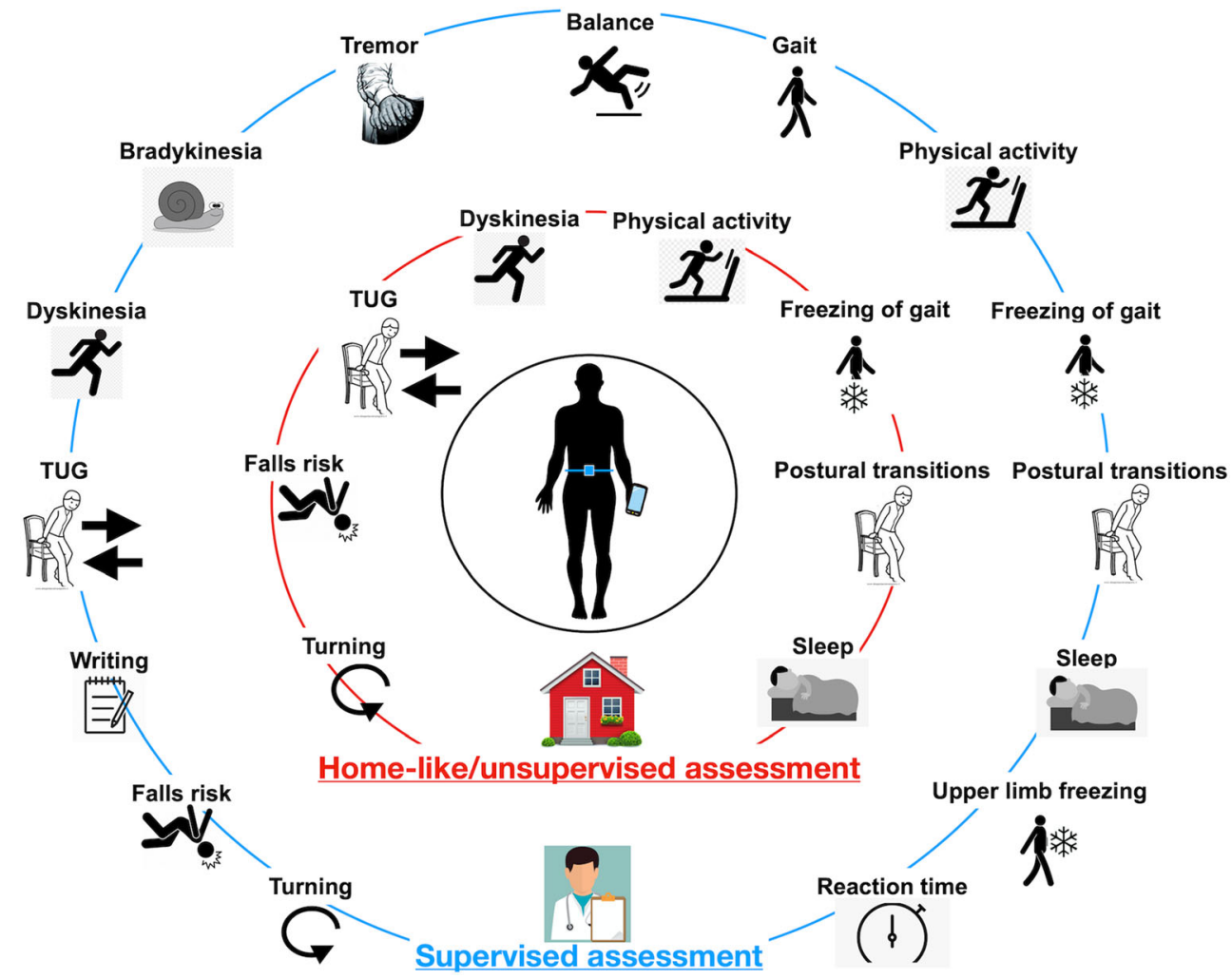


with movement disorders [24]. However, obstacles remain on the application of mHealth in clinical trials.

To analyze the key factors for successful integration of mHealth in clinical trials according to the MDS Task Force on Technology indications, we applied a 5-question quality appraisal tool for every trial that specified the mHealth employed.

1) Validation is the first requirement for the acceptable use of mHealth in clinical trials. Psychometric properties such as sensitivity, accuracy, and reliability must be already tested in the population of interest to consider mHealth a validated tool. We found that almost $90 \%$ of trials used an already validated mHealth, thus adhering to this key aspect of mHealth deployment. A higher precision provided by mHealth allows a greater signal-to-noise ratio, leading to a decrease in the number of patients required for clinical trials [5]. However, this assumption is valid when mHealth is employed as a primary outcome measure, and we observed that this happened in fewer than half of all trials and in less than $20 \%$ of the phase 3 trials needed for regulatory approval. This limitation could be partly related to regulatory barriers since robust validation (which may go, at least in some cases, far beyond the actual validation state of mHealth used in the trials evaluated here) for target endpoint and population are requested by EMA and FDA in order to use mHealth as primary outcome measures in trials for registration of new drugs.

2) An observed critical pitfall is the lack of use of mHealth for capturing multiple constructs of interest to obtain a global measure of disease-associated functional impairment or disability [4]. Our results indicate that about $60 \%$ of current trials used mHealth to collect data from $>1$ construct of interest (in most cases, 2 or 3 constructs related to motor functions). Further efforts are needed for mHealth to capture multi-dimensional data, congruent with the high complexity of patients with movement disorders. In fact, current low-cost technology allows multidimensional measures with a low burden for patients and doctors.

Our exploratory analysis showed that the integration into a single platform of different proprietary algorithms on data from a smartphone/tablet and a single inertial sensor worn at the trunk could allow the monitoring of at least 15 different PD constructs of interest, both in supervised and in unsupervised settings. We focused our exploratory analysis on a smartphone and a single inertial sensor worn at the trunk because we considered it the lessinvasive, but most comprehensive way to collect many constructs of interest simultaneously. This approach has the promise of feasible usability also at the patients' home and during daily life. Still, relevant gaps remain in the assessment of non-motor features frequently associated with movement disorders, such as cognitive dysfunction and neurobehavioral symptoms. A recent scoping review on mobile devices for cognitive assessment of older adults highlighted the role of smartphones and tablets for more efficient dementia screening at a large scale [25]. Also, several studies have focused on the evaluation of depression and anxiety through automated artificial intelligence-simulated conversations (chabots) provided by smartphone apps or non-mobile technologies or through changes in physical activity measures obtained by wearable motion sensors [26, 27]. Further studies would need to test the application of these strategies to neurological populations.

3) Another critical aspect preventing the application of mHealth as primary or secondary outcome measures in clinical trials is the uncertainty regarding the clinical relevance of data captured. Noteworthy, almost $90 \%$ of trials in Clinicaltrials.gov used mHealth with the potentiality of capturing clinically meaningful data.

4) Device feasibility and acceptability from patients and researchers can be a limiting factor in the adoption of mHealth. We found that an acceptable percentage of trials (79\%) used mHealth with a formal assessment of these features, which is worth to be considered in the application of mHealth, not only for clinical trials but also for the daily life monitoring.

5) Finally, the correlation between data captured and patient-centered outcome measures is pivotal to link with patients' activities of daily living or quality of life [28]. Such correlation should be pursued in mHealth measures to enhance the relevance of quantitative, objective measures. In this analysis, we confirmed a correlation between data captured by mHealth and patient-centered outcome measures in about $70 \%$ of trials. Though this number is quite high, more data supporting the relationship between mHealth measures and patient-centered relevant clinical endpoints are needed for their implementation as primary and secondary outcome measures in clinical trials. The use of different sensors and methodologies and the low or absent use of mHealth for many relevant constructs of interest in movement disorders, such as gait, postural instability, and non-motor symptoms, are important limitations. Construct validity, acceptability, and clinical relevance (including definition of test-retest reliability and minimal clinically relevant change) of mHealth should be validated on large populations by multicenter studies sharing the same methodology $[29,30]$. This aspect would facilitate not only the validation of mHealth for monitoring of symptoms but also for discovering features that could be used as predictors of clinical outcomes. 
There is still an important need for evidence of clinical meaningfulness of digital measures for regulatory decisions. This is a pivotal step for FDA and EMA registrations and acceptance of new digital measures in clinical trials [31]. As recently highlighted from the Clinical Trials Transformation Initiative, the selection of mHealth for data capture must occur after the clinical outcome is clearly identified (www.ctticlinicaltrials.org/files/novelendpoints-recs.pdf). mHealth objectively measure data for endpoints not previously considered or encompassed by older but more established scales. As an example, the neuroQWERTY software, which can monitor fine motor function while using a computer or smartphone, is under review by the FDA for clearance [32]. More recently, the EMA approved another mHealth mobility parameter measured in the home environment, namely stride velocity, as a secondary endpoint for baseline assessments in Duchenne muscular dystrophy studies (https://www.ema. europa.eu/en/documents/scientific-guideline/qualificationopinion-stride-velocity-95th-centile-secondary-endpointduchenne-muscular-dystrophy_en.pdf). These novel endpoints have the potential to provide high-quality data of outcomes that are meaningful to patients while theoretically enabling larger trials with reduced barriers to participation, thus making possible more sensitive, generalizable, and patient-centric assessments.

Some limitations are important to note regarding this analysis. First, most (but not all) of the ongoing clinical trials may have been identified. Other trials, particularly non-sponsored ones, may be registered in other databases or not registered at all. Thus, our findings offer only an estimation of the use of $\mathrm{mHealth}$ in ongoing trials. Second, many trials acknowledged the use of sensors or other mHealth, without specifying the exact type of technology employed; we partially solved this problem by contacting the trial contact person, but we obtained further information in only $56 \%$ of cases. Third, we focused our search on the most common movement disorders; trials on other movement disorders, such as tics and ataxias, were not considered in this study.

Finally, it is important to note that the validation, defined as the determination of the degree of validity of a measuring device, particularly when applied to mHealth is truly not a "yes or no" judgment, but rather a process, for which many factors, including the context, are relevant. Indeed, the disease stage, the variability in the phenomenology, and the possible gender, age, genetic, ethnic, and biological differences among patients represent a large source of heterogeneity threatening any process of validation. Another important consideration is that technology-based measures for any disease-related symptom would be expected to be validated on high numbers of patients diagnosed with the same disease applying the same diagnostic criteria, enrolling patients at every stage of the disease, and in many centers worldwide. Conversely, if the tool is "validated" for a population, its ability to capture nuanced data for an individual is reduced or may be misleading (as when applied to individuals without the symptom the technology was validated for).

Limitations notwithstanding, our findings suggest that mHealth are poised to gain relevance as outcome measures in clinical trials for movement disorders, even if they are currently underutilized and largely relegated to phase 2 trials as secondary or tertiary outcome measures. Efforts toward greater correlation with patient-centered outcomes and integration of technologies into a single platform will help with extending the deployment of mHealth in clinical trials to increase their signal-to-noise ratio and reduce their costs.

Required Author Forms Disclosure forms provided by the authors are available with the online version of this article.

\section{References}

1. Artusi CA, Mishra M, Latimer P, et al. Integration of technologybased outcome measures in clinical trials of Parkinson and other neurodegenerative diseases. Parkinsonism Relat Disord 2018;46: S53-S56.

2. FitzGerald JJ, Lu Z, Jareonsettasin P, Antoniades CA. Quantifying Motor Impairment in Movement Disorders. Front Neurosci 2018;12:202.

3. Jalloul N. Wearable sensors for the monitoring of movement disorders. Biom J 2018;41:249-253.

4. Merola A, Sturchio A, Hacker S, et al. Technology-based assessment of motor and nonmotor phenomena in Parkinson disease. Expert Rev Neurother 2018;18:825-845.

5. Espay AJ, Hausdorff JM, Sánchez-Ferro Á, et al. A roadmap for implementation of patient-centered digital outcome measures in Parkinson's disease obtained using mobile health technologies. Mov Disord 2019;34:657-663.

6. Espay AJ, Bonato P, Nahab FB, et al. Technology in Parkinson's disease: Challenges and opportunities. Mov Disord 2016;31:12721282.

7. Lin PC, Chen KH, Yang BS, Chen YJ. A digital assessment system for evaluating kinetic tremor in essential tremor and Parkinson's disease. BMC Neurol 2018;18:25.

8. Mitsi G, Mendoza EU, Wissel BD, et al. Biometric Digital Health Technology for Measuring Motor Function in Parkinson's Disease: Results from a Feasibility and Patient Satisfaction Study. Front Neurol 2017;8:273.

9. Lopane G, Mellone S, Corzani M, et al. Supervised versus unsupervised technology-based levodopa monitoring in Parkinson's disease: an intrasubject comparison. J Neurol 2018;265:1343-1352.

10. Pham MH, Warmerdam E, Elshehabi M, et al. Validation of a Lower Back "Wearable"-Based Sit-to-Stand and Stand-to-Sit Algorithm for Patients With Parkinson's Disease and Older Adults in a Home-Like Environment. Front Neurol 2018;9:652.

11. Ozinga SJ, Machado AG, Miller Koop M, Rosenfeldt AB, Alberts JL. Objective assessment of postural stability in Parkinson's disease using mobile technology. Mov Disord 2015;30:1214-1221.

12. Zago M, Sforza C, Pacifici I, et al. Gait evaluation using inertial measurement units in subjects with Parkinson's disease. J Electromyogr Kinesiol 2018;42:44-48.

13. Weiss A, Herman T, Giladi N, Hausdorff JM. Objective assessment of fall risk in Parkinson's disease using a body-fixed sensor worn for 3 days. PLoS One 2014;9:e96675. 
14. Ahlrichs $\mathrm{C}$, Samà $\mathrm{A}$, Lawo $\mathrm{M}$, et al. Detecting freezing of gait with a tri-axial accelerometer in Parkinson's disease patients. Med Biol Eng Comput 2016;54:223-233.

15. Popovic MB, Dzoljic E, Kostic V. A method to assess hand motor blocks in Parkinson's disease with digitizing tablet. Tohoku J Exp Med 2008;216:317-324.

16. Heremans E, Nackaerts E, Broeder S, Vervoort G, Swinnen SP, Nieuwboer A. Handwriting Impairments in People With Parkinson's Disease and Freezing of Gait. Neurorehabil Neural Repair 2016;30:911-919.

17. Pham MH, Elshehabi M, Haertner L, et al. Validation of a Step Detection Algorithm during Straight Walking and Turning in Patients with Parkinson's Disease and Older Adults Using an Inertial Measurement Unit at the Lower Back. Front Neurol 2017;8:457.

18. Lamont RM, Daniel HL, Payne CL, Brauer SG. Accuracy of wearable physical activity trackers in people with Parkinson's disease. Gait Posture 2018;63:104-108.

19. Bhidayasiri R, Sringean J, Taechalertpaisarn P, Thanawattano C. Capturing nighttime symptoms in Parkinson disease: Technical development and experimental verification of inertial sensors for nocturnal hypokinesia. J Rehabil Res Dev 2016;53:487-498.

20. Lai B, Sasaki JE, Jeng B, Cederberg KL, Bamman MM, Motl RW. Accuracy and Precision of Three Consumer-Grade Motion Sensors During Overground and Treadmill Walking in People With Parkinson Disease: Cross-Sectional Comparative Study. JMIR Rehabil Assist Technol 2020;7:e14059.

21. Del Din S, Galna B, Godfrey A, et al. Analysis of Free-Living Gait in Older Adults With and Without Parkinson's Disease and With and Without a History of Falls: Identifying Generic and DiseaseSpecific Characteristics. J Gerontol A Biol Sci Med Sci 2019;74: 500-506.

22. Warmerdam E, Hausdorff JM, Atrsaei A, et al. Long-term unsupervised mobility assessment in movement disorders. Lancet Neurol 2020

23. Cerreta F; European Medicines Agency Geriatric Expert Group. New harmonized considerations on the evaluation instruments for baseline characterization of frailty in the European Union. Br J Clin Pharmacol 2019

24. Hansen C, Sanchez-Ferro A, Maetzler W. How Mobile Health Technology and Electronic Health Records Will Change Care of Patients with Parkinson's Disease. J Parkinsons Dis 2018;8:S41S45.

25. Koo BM, Vizer LM. Mobile Technology for Cognitive Assessment of Older Adults: A Scoping Review. Innov Aging 2019;3:igy038.

26. Fleming T, Bavin L, Lucassen M, Stasiak K, Hopkins S, Merry S. Beyond the Trial: Systematic Review of Real-World Uptake and Engagement With Digital Self-Help Interventions for Depression, Low Mood, or Anxiety. J Med Internet Res 2018;20:e199.

27. Seppälä J, De Vita I, Jämsä T, et al. Mobile Phone and Wearable Sensor-Based mHealth Approaches for Psychiatric Disorders and Symptoms: Systematic Review. JMIR Ment Health 2019;6:e9819.

28. Frank L, Basch E, Selby JV; Patient-Centered Outcomes Res Insti. The PCORI perspective on patient-centered outcomes research. JAMA 2014;312:1513-1514.

29. Geritz J, Maetzold S, Steffen M, et al. Motor, cognitive and mobility deficits in 1000 geriatric patients: protocol of a quantitative observational study before and after routine clinical geriatric treatment - the ComOn-study. BMC Geriatr 2020;20:45.

30. Erb MK, Karlin DR, Ho BK, et al. mHealth and wearable technology should replace motor diaries to track motor fluctuations in Parkinson's disease. NPJ Digit Med 2020;3:6.

31. Coran P, Goldsack JC, Grandinetti CA, et al. Advancing the Use of Mobile Technologies in Clinical Trials: Recommendations from the Clinical Trials Transformation Initiative. Digit Biomark 2019;3: 145-154.

32. Matarazzo M, Arroyo-Gallego T, Montero P, et al. Remote Monitoring of Treatment Response in Parkinson's Disease: The Habit of Typing on a Computer. Mov Disord 2019;34:1488-1495.

Publisher's Note Springer Nature remains neutral with regard to jurisdictional claims in published maps and institutional affiliations. 